\title{
Effects of whole body vibration on spinal proprioception in healthy individuals
}

\author{
D.H.K. Chow ${ }^{\mathrm{a}, *}$, T.Y. Lee ${ }^{\mathrm{b}}$ and M.H. Pope ${ }^{\mathrm{c}}$ \\ ${ }^{a}$ Department of Health \& Physical Education, The Education University of Hong Kong, Tai Po, \\ New Territories, Hong Kong \\ ${ }^{\mathrm{b}}$ Department of Biomedical Engineering, The Hong Kong Polytechnic University, Hong Kong \\ ${ }^{\mathrm{c}}$ Department of Occupational Medicine, University of Aberdeen, Aberdeen, Scotland, UK
}

Received 4 July 2017

Accepted 5 March 2018

\begin{abstract}
.
BACKGROUND: Low back pain (LBP) is a common health problem with high reoccurrence rate. As patients with LBP are often found to be proprioception impaired, new proprioception exercises should be explored. Whole body vibration (WBV) has been proven to improve muscle function and proprioception.

OBJECTIVE: The aim of this study was to determine the effects of WBV on spinal proprioception when WBV was administered in standing and seated postures.

METHODS: Twenty healthy male individuals (mean age: $23.2 \pm 1.2$ years) were recruited and randomly assigned to two WBV groups: WBV in standing or WBV in seated posture. Their body posture, lumbar repositioning ability, maximum reaching distance and lumbopelvic coordination during dynamic motion in flexion and extension were assessed before, immediately after, 30 minutes after and 1 hour after 5 minutes of WBV (18 Hz, $6 \mathrm{~mm}$ amplitude) exposure. A Mixed ANOVA was used to analyze the effects of group and time factors on these four outcome measures.

RESULTS: There were no significant interaction (group and time) and group effects on all outcome measures. Participants were found to have significant different time effect on body posture, lumbar repositioning ability, maximum reaching distance and lumbopelvic coordination.

CONCLUSIONS: WBV could significantly improve spinal proprioception including body posture, lumbar repositioning ability, maximum reaching distance and lumbopelvic coordination in healthy individuals. WBV protocol is recommended to confirm its clinical application for improving spinal proprioception and its effects on patients with LBP is warranted.
\end{abstract}

Keywords: Body posture, proprioception, lumbar spine, low back pain

\section{Introduction}

Low back pain (LBP) is a common health problem worldwide and has been treated in the primary healthcare units [1]. This musculoskeletal problem appears with lifetime prevalence around 50-70\%, with $80 \%$ of patients classified as non-specific LBP [2]. As the

\footnotetext{
*Address for correspondence: Prof. Daniel H.K. Chow, Department of Health \& Physical Education, The Education University of Hong Kong, Tai Po, New Territories, Hong Kong. Tel.: +852 2948 6241; Fax: +852 2948 7853; E-mail: danielchow@eduhk.hk.
}

etiology is still unknown [3], most treatments for patients with LBP are not that effective [4-6].

Spinal proprioception is a feedback mechanism which facilitates spine positioning and movement [7]. Proprioception of patients with LBP is significantly impaired in lumbopelvic movement pattern, repositioning accuracy and balance ability [8-11]. Proprioceptive deficit would cause delayed protective muscle reflexes and coordination, hence muscle contraction would not respond fast enough for excessive joint movement to protect the spine from injury 
[10]. Excessive loading would be transmitted to joint surfaces and induce more pain to the patient with LBP, causing high reoccurrence rate [1]. Proprioceptive training is therefore recommended for patients with LBP [12].

Although whole body vibration (WBV) has been implicated in the causation of LBP, at frequencies of 4-6 Hz [13], higher frequencies of WBV have been used as training exercises for athletics and healthy people $[14,15]$. WBV generated either by vertical or rotational sinusoidal vibration could stimulate proprioceptive receptors such as muscle spindles and joint mechanoreceptors $[16,17]$. As muscle strength and balance could be improved by WBV [18, 19] and muscle stiffness and joint stability could be modified by mechanoreceptor activity through gamma efferent stimulation [20], WBV has potential for delivering proprioception training $[21,22]$.

WBV of $6 \mathrm{~mm}$ displacement at $18 \mathrm{~Hz}$ with progressive duration for 3 consecutive months has been demonstrated to relieve pain in patients with LBP [20]. Repositioning ability was also improved in pelvic tilting while standing, after 5 minutes $18 \mathrm{~Hz}-$ $4 \mathrm{~mm}$ WBV in normal individuals [22]. However, effects of WBV on dynamic motor control of the lumbar spine have not been investigated. Spinal proprioception was assessed using the Dynamical Systems Theory approach in terms of functional spinal coordination and stability [23, 24]. As attenuation of WBV has been found to occur at the knee level when the frequency of vibration is larger than $15 \mathrm{~Hz}$ [25], transmissibility of the vibration would be likely affected [26] if the participants adopt a standing posture during WBV. As application of WBV in seated posture might deliver vibration to the spinal region with less attenuation, the effects of WBV administered in these two postures were therefore, compared in this study.

Based on the rationale of above-mentioned Dynamical Systems Theory approach, this study used body posture, seated repositioning ability, maximum reaching distance and lumbopelvic coordination to represent spinal proprioception. The hypothesis was that the improvement of spinal proprioception under WBV could be validated by the observations of these four outcome measures.

\section{Methods}

In this study, effects of standing and seated WBV on spinal proprioception were investigated in healthy individuals. Body posture, seated repositioning ability, maximum reaching distance and lumbopelvic coordination were assessed before WBV as well as immediately, 30 minutes and 1 hour after WBV. Twenty healthy male participants including college students and workers were recruited and randomly divided into standing group and seated group for receiving WBV in standing or seated posture, respectively. The sample size was figured out by $\mathrm{G}^{*}$ Power software for Mixed ANOVA statistical test with significant level at $p=0.05$ and statistical power of 0.8 . The mean (SD) age, body height and weight of the participants in standing group were 23.2 (1.2) years, $172.1(6.3) \mathrm{cm}$ and $63.2(3.9) \mathrm{kg}$, respectively. For the seated group participants, their mean (SD) age, body height and weight were 21.0 (1.1) years, 171.7 (4.3) $\mathrm{cm}$ and $59.8(5.3) \mathrm{kg}$, respectively. Ethical approval was obtained from the Human Ethics Committee of the Education University of Hong Kong and written informed consent from all of the participants was obtained before the study. Participants in healthy condition, with regular exercises (at least 150 minutes of moderate aerobic activity every week) and age between 18-30 years were included, and those with chronic non-specific low back pain either continuously for more than 6 months or intermittently for more than 2 years, any known musculoskeletal, inner ear, or neurological disorders diagnosed by doctor, as well as drug and steroid users were excluded.

WBV was delivered using a Galileo sport (teeterboard-like) platform (Novetec, Pforzheim, Germany). Participants in the standing group underwent WBV at $18 \mathrm{~Hz}, 6 \mathrm{~mm}$ amplitude for 5 minutes while maintaining a neutral standing posture on the vibrating platform with knees slightly flexed. Amplitude was adjusted to $6 \mathrm{~mm}$ by setting foot distance at $33 \mathrm{~cm}$ apart and equidistant from the central axis. Both feet were positioned in parallel on the platform [22]. The participants were allowed to hold the hand bar of the platform if needed (Fig. 1).

Participants in the seated group were asked to sit in a relaxed posture on the WBV platform with their hands placed comfortably on the thigh (Fig. 1). Participants in both groups were asked to gaze horizontally during the vibration [22].

Retro-reflective markers were affixed to the participants with the body slightly flexed to minimize the effects of skin traction during motion [27]. The markers were attached to the chin, C7, T2, T5, T7, T12, L1, L3, S1, bilateral anterior and posterior superior iliac spines and the right hand ulnar styled process after palpation. Markers were also attached to greater 


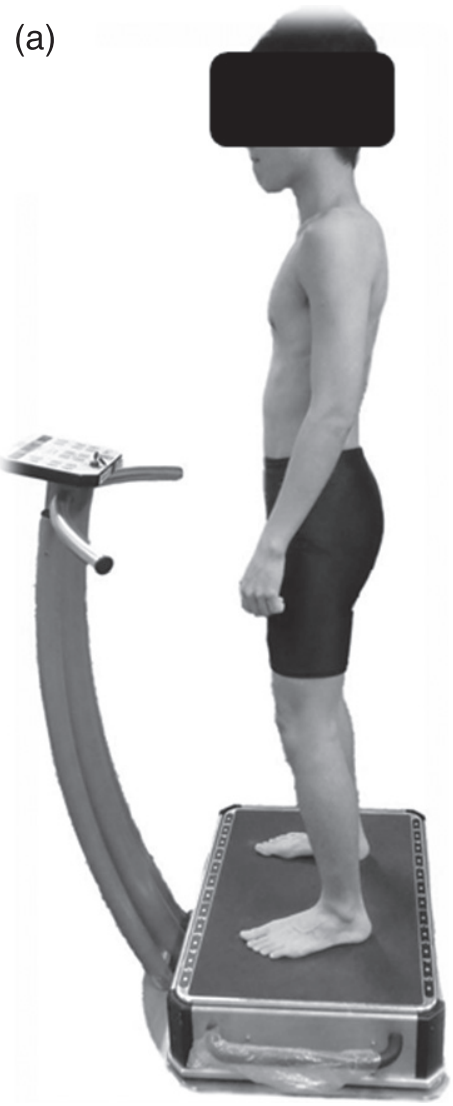

(b)

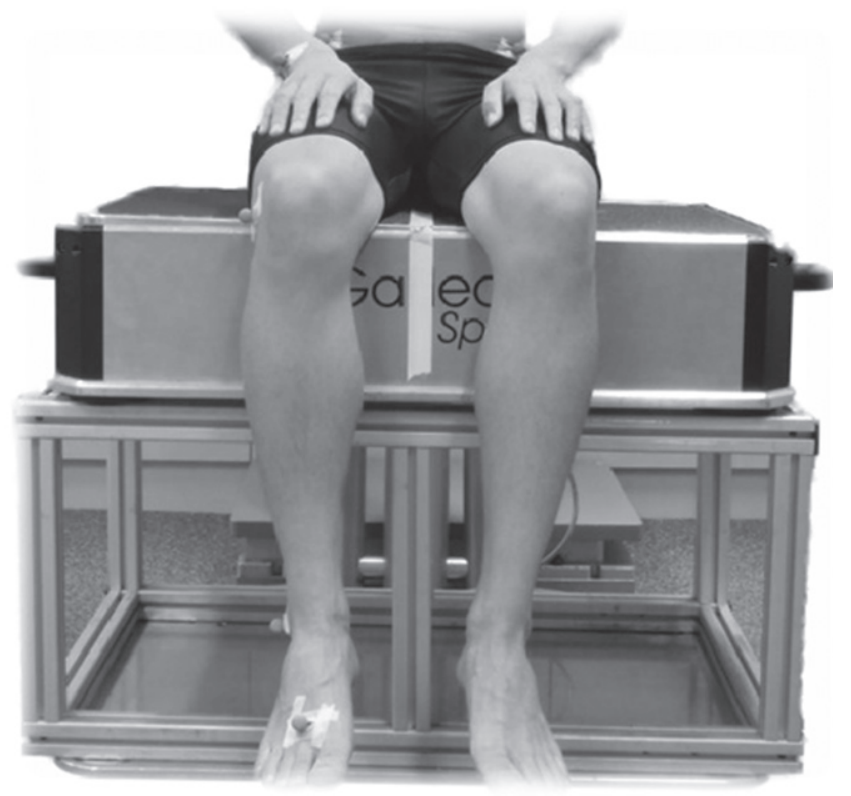

Fig. 1. Subject under whole body vibration in (a) standing and (b) seated postures.

trochanter, lateral condyle, lateral malleolus, second metatarsal head and heel of the right leg (Fig. 2). Surgical tape was used to further secure the markers during WBV. Relative movement between skin markers and the bony structure beneath was neglected. An eight-camera motion analysis system (Vicon Nexus, MXF40 cameras, Oxford Metric, UK) was used to monitor positions of the markers. All data were sampled at $100 \mathrm{~Hz}$ and low-pass filtered at $3 \mathrm{~Hz}$ [28].

Back curvature and lower limb posture of the participants were measured before and after WBV (Fig. 3). Participants were asked to perform a body posture task in normal upright stance with hands rested aside and feet separated at a comfortable distance during the measurement. Feet position was marked on the floor and participants were asked to keep their gaze at a target positioned $2 \mathrm{~m}$ in front at individuals' eye level. Totally, 6 measurement trials were conducted and the data were averaged for data analysis. Participants were asked to walk around a 6-m loop between consecutive trials for the repeatability issue [29].
For the seated repositioning error test, participants were blindfolded and sat on a rigid stool with arms on the thighs in a relaxed manner [10]. Participants were asked to reproduce a criterion position, i.e. the initial neutral upright posture, five times following lumbar flexion. Repositioning error was defined as the mean difference in lumbar flexion angle relative to the pelvis between the five trials and the criterion position. Repositioning error was expressed in terms of absolute error, variable error and constant error for denoting the error in direction, consistency and magnitude, respectively [11].

Maximum reaching distance of the participants in performing forward reaching task was assessed since it could assess body balance and characterizing lumbopelvic movement based on Dynamical Systems Theory [30]. Prior to the assessment, mean maximum reach distance of each participant was determined. Participants were asked to slide the bar on a yardstick set at shoulder height as far as possible without taking a step or losing balance for three repetitions. Half of the maximum reaching distance was then set as 

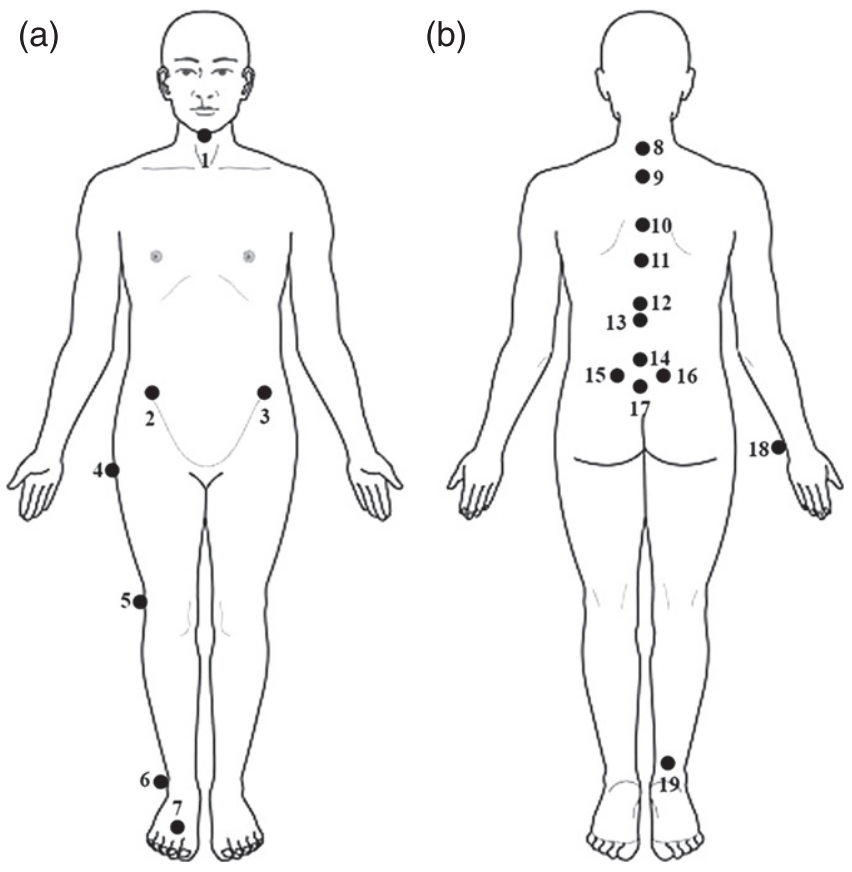

\begin{tabular}{|c|c|}
\hline Chin & 1 \\
\hline Anterior Superior lliac Spine & 2,3 \\
\hline Great Trochanter & 4 \\
\hline Lateral Condyle & 5 \\
\hline Lateral Malleolus & 6 \\
\hline Second Metatarsal Head & 7 \\
\hline C7 & 8 \\
\hline T2 & 9 \\
\hline T5 & 10 \\
\hline T7 & 11 \\
\hline T12 & 12 \\
\hline L1 & 13 \\
\hline L3 & 14 \\
\hline Posterior Superior lliacSpine & 15,16 \\
\hline S1 & 17 \\
\hline UInar styled process & 18 \\
\hline Heel & 19 \\
\hline
\end{tabular}

Fig. 2. Makers attachment (a) front view and (b) back view.

the target reaching point for the subsequent dynamic reaching test. In the test, participants were asked to reach for the target reaching point using the trunk and pelvis as if reaching over a cupboard without taking a step over a period of $3 \mathrm{~s}$, and then restore the upright position in the following $3 \mathrm{~s}$ with the aid of a metronome. The $3 \mathrm{~s}$ duration was adopted to standardize the motion in an appropriate speed as described in previous study [30]. Three cycles were performed for each trial and three warm-up trials were given before the data of the fourth trial were used for data analysis.

To assess the lumbopelvic coordination, angular displacements of the lumbar spine and pelvis in each forward and backward motion were time-normalized to 120 points [8]. Phase angles were calculated as the arc tangent of velocity of each body segment divided by its own displacement. Continuous relative phase curves were derived from the phase difference between the lumbar spine and the pelvis [30, 31]. To quantify the change in continuous relative phase curves, two parameters, namely mean absolute relative phase (MARP) and deviation phase in the forward and backward directions, were calculated using the equations proposed by Chow et al. [31]. MARP was the average of the relative phase values over the continuous relative phase curve and deviation phase was the average standard deviations of

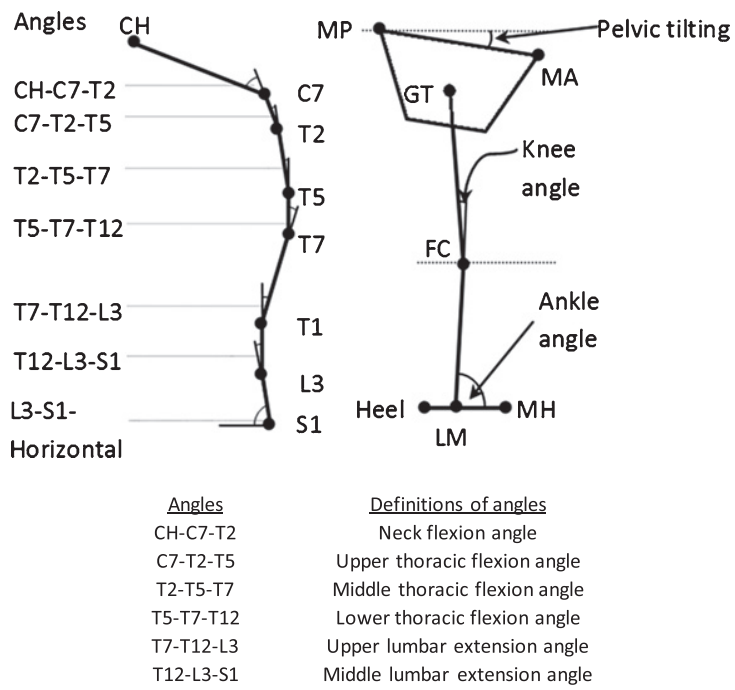

Fig. 3. Definitions of various angles of body alignment (Left: Spinal curvature; Right - lower limb angles). $\mathrm{CH}=$ chin; $\mathrm{MP}=$ mid-point of posterior superior iliac spines; $\mathrm{MA}=$ mid-point of anterior superior iliac spine; $\mathrm{GT}=$ great trochanter; $\mathrm{FC}=$ femoral condyle; $\mathrm{LM}=$ lateral malleolus; $\mathrm{MH}=$ second metatarsal head.

the ensemble continuous relative phase curve points [31]. These parameters were used to quantify the phasic relationship between the two segments of interest and pattern stability throughout the reaching process. Smaller MARP represented more in-phase 
lumbopelvic coordination while smaller deviation phase represented more stable movement pattern and vice-versa. Smaller MARP and more in-phase indicated the improvement in lumbopelvic coordination that might be beneficial to health status in terms of better balance control during dynamical motion.

As symmetric motion was investigated in the study, only angular motion in the sagittal plane was analyzed. Independent $t$-test was used to compare the demographic data between the two groups. Mixeddesign analysis of variance (Mixed ANOVA) was used to study the changes in body posture, maximum reaching distance, repositioning ability and lumbopelvic coordination between the standing and seated WBV groups (i.e. group factor) before and after WBV (i.e. time factor). Statistical software (IBM SPSS Statistics 21, Inc., Chicago. IL, IBM, USA) was used for data analysis with level of significance set at $p=0.05$ and least significant difference (LSD) criterion was adopted for post-hoc comparisons.

\section{Results}

Pooled means and standard deviations of all outcome measures and all statistical results of Mixed ANOVA were computed (Table 1).

There was no significant difference in body weight, age and height between the two testing groups (Body weight: $p=0.870$; Age: $p=0.096$; Height: $p=0.113$ ).

For the body posture, there were no significant interaction between group and time factors $(p=0.607)$ and main group factor $(p=0.733)$ in lower lumbar extension angle. However, there was a significant main effect of time $(p=0.030)$ for the WBV where a significant decrease in lower lumbar lordosis immediately after WBV was found (Table 1). The lumbar extension angle was then increased back to a value that was not significant different with that of before WBV. For pelvis tilting, there were no significant interaction between group and time factors $(p=0.295)$ and main group factor $(p=0.987)$. Significant main effect of time $(p=0.018)$. There was a significant increase in pelvis tilting immediately after WBV (Table 1). The pelvis tilting was then decreased back to a value that was not significant different with that of before WBV. No significant changes in other parameters for repositioning ability were observed.

For lumbar repositioning ability, there was no significant interaction between group and time factors in absolute error $(p=0.107)$, no significant main effect of group ( $p=0.818)$ and significant main effect of time $(p=0.009)$ (Table 1). Absolute error was found to reduce immediately and 30 minutes after WBV (Table 1).

For maximum reaching distance, there was no significant interaction between group and time factors $(p=0.952)$. Changes in maximum reaching distance over time were not significantly different between the two groups $(p=0.139)$. There was a significant main effect of time $(p<0.001)$ for the WBV, maximum reaching distance was found to have significant change with time, i.e. it increased immediately after WBV, and this increment maintained 30 minutes and 1 hour after WBV (Table 1).

For lumbopelvic coordination: MARP in the forward direction, no significant interaction was found between group and time factors $(p=0.403)$. There was no significant main effect of group $(p=0.330)$. There was a significant main effect of time $(p<0.001)$ with a significant decrease in MARP immediately, 30 minutes and 1 hour after WBV compared to that before WBV (Table 1). There was no significant change in deviation phase after WBV for both groups in the forward direction.

For lumbopelvic coordination: For MARP in the backward direction, there was no significant interaction between group and time factors $(p=0.962)$. There was no significant main effect of group $(p=0.671)$. There was a significant main effect of time $(p<0.001)$ with a significant decrease in MARP immediately, 30 minutes and 1 hour after WBV (Table 1). There was a significantly increase in MARP 1 hour after WBV in comparison with that of immediately after WBV and it was still lower than that of before WBV (Table 1). There was no significant change in deviation phase after WBV for both groups in the backward direction.

\section{Discussion}

Three subsystems, which are the active musculoskeletal, passive musculoskeletal and neural feedback subsystems, are required for maintaining spinal stability [32]. Mechanical receptors of the neural feedback subsystems located in the muscles and tendons are essential for providing proprioception for spinal motor control by cooperating with the visual and vestibular systems to provide dynamic updates of self-orientation related to the surroundings during locomotion and navigation [3]. Hence, different tests were performed in this study to 
Table 1

Summary of statistical results of the Mixed ANOVA and pooled means and standard deviations of all outcome measures

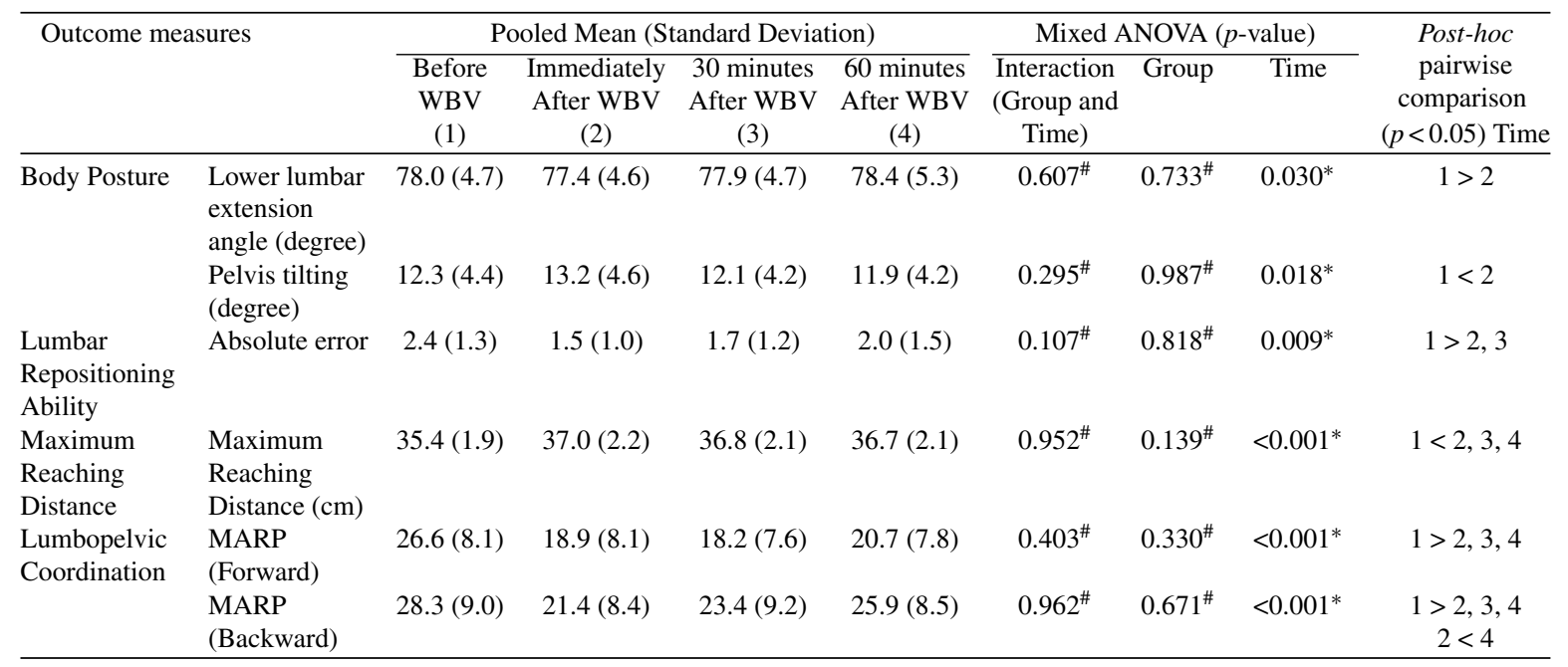

${ }^{*}$ Significant effect $(p<0.05) .{ }^{\#}$ Non significant $(p<0.05)$.

thoroughly investigate the effects of WBV on spinal proprioception.

Significant change in body posture was found before and after WBV. There was a significant decrease in lower lumbar lordosis after WBV. This would probably be due to the increase of flexibility of the muscle and the virtual muscle lengthening effect of the WBV [33], which is likely due to the stimulation of the primary ending of the muscle spindles by the vibration [34]. Besides, pelvis tilting was increased significantly immediately after WBV and no other improvement on body posture was found.

For the repositioning test, participants performed the repositioning test in a seated posture so as to minimize the afferent input from the lower limb [35]. There was significant improvement in lumbar repositioning ability after WBV. The beneficial effect of WBV on lumbar repositioning ability has been reported in normal subjects [22].

Reaching tasks are necessary for daily activities $[36,37]$. These tasks generally involve reaching forward and grasping objects in different heights, and returning to an upright position [37]. The dynamic reaching test was used to assess postural stability and activity of the lower limb and trunk muscles [35], and hence were related to the ability of dynamic balance and postural control of an individual that might be beneficial to the improvement of spinal proprioception. A significant increase in maximum reaching distance after WBV suggested that there was an improvement in balance and postural control after WBV. Interestingly, this effect persisted for 1 hour after WBV.

MARP in the forward direction was found to be significantly reduced immediately after WBV, which showed that the lumbar and pelvic regions were moving in a more coordinated way after WBV. This might be caused by the repetitive WBV mechanical stimulation, leading to a rearrangement of postural control strategies [38] and indicate a beneficial effect of WBV. However, it was observed that the effects of WBV on lumbopelvic coordination were more apparent in the forward direction than in the backward direction. This suggested that WBV might have a stronger effect on back muscles [39]. This might explain the improvement of motor function and pain relief in LBP patients after receiving 12-week WBV treatment [21]. The reduced MARP indicated a rearrangement effect in the lumbar and pelvic regions, and might explain the beneficial effect of WBV. Deviation phase has been used as an indicator for reflecting the stability of the motion pattern. As only normal healthy participants were tested in this study, it was not surprising that this parameter was not significantly affected after WBV. It would be interesting to know whether this will be affected in patients with LBP or not.

When mechanoreceptors such as the muscle spindles are activated by WBV, the $\alpha$-motor neurons are also activated, leading to muscle contractions 
similar to the tonic vibration reflex [15]. Similar to the findings of other studies, WBV could improve neuromuscular function [40] and increase muscle flexibility [41-43] and so improve maximum reaching distance and lumbo-pelvic coordination. The improvement is likely to be due to activation of the Ia-afferent fibers and $\alpha$-motor neurons by repetitive stimulation of the back muscles during WBV [44], causing an increment in flexibility. It has also been hypothesized that the increase of muscle flexibility and improvement of proprioception would likely be due to an increased number of type II muscle fibre recruitment, with the absence of muscle fatigue or overexertion [45]. In addition, the impact of vibratory stimulation on central motor command may activate specific areas of the brain as well as influence motor command and facilitate the dynamic motion [45]. Another possible explanation for the increase in muscle flexibility was an increase of blood flow in muscles after WBV [46], which would lead to an increase in muscle temperature, and hence, muscle extensibility [47]. However, this phenomenon was observed in a period much longer than the duration of the WBV application in our study.

Improvement in neuromuscular performance and muscle flexibility has also been observed after static stretching [48, 49]. However, the increase in flexibility might likely be due to the stretch tolerance more than the modification of the muscle viscoelastic properties. Passive warm-up or voluntary exercises could not recruit type II muscle fibres in the working muscles, but WBV could, due to the facilitation of neuromuscular coordination by the stimulus [50]. WBV may be effective for recruiting motor neurons and activating large amounts of muscle [50]. This was achieved by providing a greater activation of the mechanoreceptors and the tonic vibration reflex, which act predominantly on $\alpha$-motor neurons [18]. Moreover, it has also been demonstrated that the effect of WBV together with stretching on knee flexibility persisted longer than stretching alone [51]. This may also occur in the spinal musculature [52].

One of the limitations of this study was the lack of investigation of long-term effects of WBV on the participants. Also, the within group effect was not considered in all statistical tests. Moreover, WBV has been shown to improve the strength of leg muscles, which might affect the results in performing dynamic reaching test. Additionally, the positive outcomes of this study may not be applicable for LBP patients since only healthy participants were invited. Moreover, the amplitude of WBV received by the participants in seated posture would vary and the vibration delivered to the head in seated posture would probably larger than standing posture. Previous studies have shown that the frequency, duration and body posture adopted during WBV could possibly lead to chronic non-specific LBP [53-55]. It is necessary to select WBV protocol very carefully so as to balance risk and benefit of WBV. It should be noted that the frequency and amplitude of the WBV is critical, because vibration could produce very different responses, either beneficial or dangerous [51].

\section{Conclusion}

It was demonstrated that 5 minutes of low frequency WBV could significantly improve proprioception in terms of lumbopelvic coordination and reaching function without any apparent adverse effect. The effects were not apparently different when WBV was administered with participants in standing or seated posture. Further study on patients with LBP who have undergone similar WBV protocol is recommended to confirm its clinical application for improving compromised proprioception.

\section{Conflict of interest}

None to report.

\section{References}

[1] Koes BW, van Tulder MW, Thomas S. Diagnosis and treatment of low back pain. BMJ. 2006;332(7555):1430-4.

[2] Croft PR, Macfarlane GJ, Papageorgiou AC, Thomas E, Silman AJ. Outcome of low back pain in general practice: A prospective study. BMJ. 1998;316(7141):1356-9.

[3] Panjabi MM. The stabilizing system of the spine. Part I. Function, dysfunction, adaptation, and enhancement. J Spinal Disord. 1992;5(4):383-9.

[4] Borman P, Keskin D, Bodur, H. The efficacy of lumbar traction in the management of patients with low back pain. Rheumatology International. 2003;23(2):82-6.

[5] Ebadi S, Henschke N, Ansari NN, Fallah E, van Tulder MW. Therapeutic ultrasound for chronic low-back pain. Cochrane Database of Systematic Reviews. 2011;3.

[6] Rubinstein SM, Terwee CB, Assendelft WJ, de Boer MR, van Tulder MW. Spinal manipulative therapy for acute lowback pain. The Cochrane Library. 2012.

[7] Taimela S, Kankaanpää M, Luoto S. The effect of lumbar fatigue on the ability to sense a change in lumbar position. A controlled study. Spine. 1999;24(13):1322-7.

[8] Silfies SP, Bhattacharya A, Biely S, Smith SS, Giszter S. Trunk control during standing reach: A dynamical system 
analysis of movement strategies in patients with mechanical low back pain. Gait Posture. 2009;29(3):370-6.

[9] Brumagne S, Lysens R, Swinnen S, Verschueren S. Effect of paraspinal muscle vibration on position sense of the lumbosacral spine. Spine. 1999;24(13):1328-31.

[10] O'Sullivan PB, Burnett A, Floyd AN, Gadsdon K, Logiudice J, Miller D, Quirke H. Lumbar repositioning deficit in a specific low back pain population. Spine. 2003;28(10):1074-9.

[11] Brumagne S, Cordo P, Lysens R, Verschueren S, Swinnen $\mathrm{S}$. The role of paraspinal muscle spindles in lumbosacral position sense in individuals with and without low back pain. Spine. 2000;25(8):989-94.

[12] Kong YS, Jang GU, Park S. The effects of prone bridge exercise on the Oswestry disability index and proprioception of patients with chronic low back pain. Journal of Physical Therapy Science. 2015;27(9):2749-52.

[13] Magnusson ML, Pope MH, Wilder DG, Areskoug B. Are occupational drivers at an increased risk for developing musculoskeletal disorders? Spine. 1996;21(6):710-7.

[14] Bosco C, Colli R, Introini E, Cardinale M, Tsarpela O, Madella A, Tihanyi J, Viru A. Adaptive responses of human skeletal muscle to vibration exposure. Clin Physiol. 1999;19(2):183-7.

[15] Rittweger J, Beller G, Felsenberg D. Acute physiological effects of exhaustive whole-body vibration exercise in man. Clin Physiol. 2000;20(2):134-42.

[16] Burke D, Hagbarth KE, Hofstedt L, Wallin BG. The responses of human muscle spindle endings to vibration of non-contracting muscles. J Physiol. 1976;261(3):673-93.

[17] Roll JP, Martin B, Gauthier GM, Mussa Ivaldi F. Effects of whole-body vibration on spinal reflexes in man. Aviat Space Environ Med. 1980;51(11):1227-33.

[18] Delecluse C, Roelants M, Verschueren S. Strength increase after whole-body vibration compared with resistance training. Med Sci Sports Exerc. 2003;35(6):1033-41.

[19] Bogaerts A, Verschueren S, Delecluse C, Claessens AL, Boonen S. Effects of whole body vibration training on postural control in older individuals: A 1 year randomized controlled trial. Gait Posture. 2007;26(2):309-16.

[20] Johansson H, Sjölander P, Sojka P. A sensory role for the cruciate ligaments. Clin Orthop Relat Res. 1991;268:16178.

[21] Rittweger J, Just K, Kautzsch K, Reeg P, Felsenberg D. Treatment of chronic lower back pain with lumbar extension and whole-body vibration exercise-a randomized controlled trial. Spine. 2002;27(17):1829-34.

[22] Fontana TL, Richardson CA, Stanton WR. The effect of weight-bearing exercise with low frequency, whole body vibration on lumbosacral proprioception: A pilot study on normal participants. Aust J Physiother. 2005;51(4):259-63.

[23] Kurz M, Stergiou N. Applied dynamic systems theory for the analysis of movement. In: Innovative analyses of human movement: Analytical tools for human movement research; 2004, pp. 93-119.

[24] Stergiou N, Harbourne R, Cavanaugh J. Optimal movement variability: A new theoretical perspective for neurologic physical therapy. J Neurol Phys Ther. 2006;30(3):120-9.

[25] Pollock RD, Woledge RC, Mills KR, Martin FC, Newham DJ. Muscle activity and acceleration during whole body vibration: Effect of frequency and amplitude. Clin Biomech. 2010;25(8):840-6.

[26] Rubin C, Pope M, Fritton JC, Magnusson M, Hansson T, McLeod K. Transmissibility of 15 -hertz to 35 -hertz vibrations to the human hip and lumbar spine: Determining the physiologic feasibility of delivering low-level anabolic mechanical stimuli to skeletal regions at greatest risk of fracture because of osteoporosis. Spine. 2003;28(23):2621-7.

[27] Swinkels A, Dolan P. Regional assessment of joint position sense in the spine. Spine. 1998;23(5):590-7.

[28] Winter A. Choice of cutoff frequency-Residual Analysis. In: Biomechanics and motor control of human movement. 2005, pp. 49-50.

[29] Chow DH, Leung KT, Holmes AD. Changes in spinal curvature and proprioception of schoolboys carrying different weights of backpack. Ergonomics. 2007;50(12):2148-56.

[30] Silfies SP, Bhattacharya A, Biely S, Smith SS, Giszter S. Trunk control during standing reach: A dynamical system analysis of movement strategies in patients with mechanical low back pain. Gait Posture. 2009;29(3):370-6.

[31] Chow DHK, Wang C, Pope MH. Effects of backpack carriage on lumbopelvic control: A dynamical systems analysis. International Journal of Industrial Ergonomics. 2014;44(4):493-8.

[32] Duncan PW, Weiner DK, Chandler J, Studenski S. Functional reach: A new clinical measure of balance. J Gerontol. 1990;45(6):192-7.

[33] Roll JP, Vedel JP, Ribot E. Alteration of proprioceptive messages induced by tendon vibration in man: A microneurographic study. Exp Brain Res. 1989;76(1):213-22.

[34] Issurin VB, Tenenbaum G. Acute and residual effects of vibratory stimulation on explosive strength in elite and amateur athletes. J Sports Sci. 1999;17(3):177-82.

[35] Rothmuller C, Cafarelli E. Effect of vibration on antagonist muscle coactivation during progressive fatigue in humans. J Physiol. 1995;485(3):857-64.

[36] Row BS, Cavanagh PR. Reaching upward is more challenging to dynamic balance than reaching forward. Clin Biomech. 2007;22(2):155-64.

[37] Huang MH, Brown SH. Age differences in the control of postural stability during reaching tasks. Gait Posture. 2013;38(4):837-42.

[38] Schuhfried O, Mittermaier C, Jovanovic T, Pieber K, Paternostro-Sluga T. Effects of whole-body vibration in patients with multiple sclerosis: A pilot study. Clin Rehabil. 2005;19(8):834-42.

[39] Zhou J, Ning X, Fathallah F. Differences in lumbopelvic rhythm between trunk flexion and extension. Clinical Biomechanics. 2016;32:274-9.

[40] Abercromby AF, Amonette WE, Layne CS, McFarlin BK, Hinman MR, Paloski WH. Variation in neuromuscular responses during acute whole-body vibration exercise. Med Sci Sports Exerc. 2007;39(9):1642-50.

[41] Van den Tillaar R. Will whole-body vibration training help increase the range of motion of the hamstrings? J Strength Cond Res. 2006;20(1):192-6.

[42] Jacobs PL, Burns P. Acute enhancement of lower-extremity dynamic strength and flexibility with whole-body vibration. J Strength Cond Res. 2009;23(1):51-7.

[43] Karatrantou K, Gerodimos V, Dipla K, Zafeiridis A. Wholebody vibration training improves flexibility, strength profile of knee flexors, and hamstrings-to-quadriceps strength ratio in females. J Sci Med Sport. 2012.

[44] Seidel H. Myoelectric reactions to ultra-low frequency and low-frequency whole body vibration. Eur J Appl Physiol. 1988;57(5):558-62.

[45] Avelar NC, Costa SJ, da Fonseca SF, Tossige-Gomes R, Gripp FJ, Coimbra CC, Lacerda AC. The effects of passive warm-up vs. Whole-body vibration on high-intensity performance during sprint cycle exercise. J Strength Cond Res. 2012;26(11):2997-3003. 
[46] Kerschan-Schindl K, Grampp S, Henk C, Resch H, Preisinger E, Fialka- Moser V, Imhof $\mathrm{H}$. Whole-body vibration exercise leads to alterations in muscle blood volume. Clin Physiol. 2001;21(3):377-82.

[47] Issurin VB, Liebermann DG, Tenenbaum G. Effect of vibratory stimulation training on maximal force and flexibility. J Sports Sci. 1994;12(6):561-6.

[48] Folpp H, Deall S, Harvey LA, Gwinn T. Can apparent increases in muscle extensibility with regular stretch be explained by changes in tolerance to stretch? Aust J Physiother. 2006;52(1):45-50.

[49] Magnusson SP, Simonsen EB, Aagaard P, Boesen J, Johannsen F, Kjaer M. Determinants of musculoskeletal flexibility: Viscoelastic properties, cross-sectional area, EMG and stretch tolerance. Scand J Med Sci Sports. 1997;7(4):195-202.

[50] Kelly SB, Alvar BA, Black LE, Dodd DJ, Carothers $\mathrm{KF}$, Brown LE. The effect of warm-up with whole-body vibration vs. cycle ergometry on isokinetic dynamometry. J Strength Cond Res. 2010;24(11):3140-3.
[51] Cardinale M, Pope MH. The effects of whole body vibration; dangerous or advantageous. Acta Physiol Hung. 2003;90(3):195-206.

[52] Feland JB, Hawks M, Hopkins JT, Hunter I, Johnson AW, Eggett DL. Whole body vibration as an adjunct to static stretching. Int J Sports Med. 2010;31(8):584-9.

[53] Pope MH, Hansson TH. Vibration of the spine and low back pain. Clinical Orthopaedics and Related Research. 1992;279:49-59.

[54] Bongiovanni LG, Hagbarth KE, Stjernberg L. Prolonged muscle vibration reducing motor output in maximal voluntary contractions in man. The Journal of Physiology. 1990;423(1):15-26.

[55] Rubin C, Pope M, Fritton JC, Magnusson M, Hansson T, McLeod K. Transmissibility of 15 -hertz to 35-hertz vibrations to the human hip and lumbar spine: Determining the physiologic feasibility of delivering low-level anabolic mechanical stimuli to skeletal regions at greatest risk of fracture because of osteoporosis. Spine. 2003;28(23):2621-7. 